\title{
Implementation of flow control over wirelessHART sensor network using wirelessHART adaptors
}

\author{
Sabo Miya Hassan ${ }^{1}$, Kishore Bingi ${ }^{2}$, Rosdiazli Ibrahim ${ }^{3}$, Lim Jin Chein ${ }^{4}$, \\ ThasarathaRao Supramaniam ${ }^{5}$ \\ ${ }^{1}$ Department of Electrical and Electronic Engineering, Abubakar Tafawa Balewa University, Nigeria \\ 2,3,4,5 Department of Electrical and Electronic Engineering, Universiti Teknologi PETRONAS, Malaysia
}

\begin{tabular}{l}
\hline Article Info \\
\hline Article history: \\
Received Oct 30, 2018 \\
Revised Feb 9, 2019 \\
Accepted Feb 25, 2019 \\
\hline
\end{tabular}

Keywords:

Adaptor

Flow process

PID control

Setpoint weighting

WirelessHART sensor network

\begin{abstract}
Despite the advantages of the industrial wireless standards such as WirelessHART, ISA100.11a and Wireless Networks for Industrial Automation-Process Automation (WIA-PA), their application still faces a lot of challenges especially when it comes to interfacing with the real plant. This is due to lack of adequate infrastructures such as interfacing circuitry to establish communication between the WirelessHART nodes and the actuators and sensors. Therefore, this paper presents the application of locally developed WirelessHART adaptors for flow process control. The adaptors serve as an interface between the WirelessHART network and the sensor and actuator of the plant. Experimental results of the controllers compared showed that wireless control is possible using the developed adaptors.
\end{abstract}

\section{Copyright (c) 2019 Institute of Advanced Engineering and Science.} All rights reserved.

\section{Corresponding Author:}

Sabo Miya Hassan,

Department of Electrical and Electronic Engineering,

Abubakar Tafawa Balewa University,

PMB 0248, Bauchi, Nigeria.

Email: smhassan@atbu.edu.ng

\section{INTRODUCTION}

Recent advances in wireless sensor networks (WSNs) have attracted keen interest by researchers [1-5]. This is because in wireless communication technology, data and information are transmitted without the need of physical medium. Therefore, the technology brings about the benefits of reduce cabling as well as space saving among others. Despite these advantages, its deployment in the monitoring and control industry was sluggish. This was mainly due to lack of suitable industrial standards and interfaces such as wireless-Adaptor and interfacing software. Recent advances in the technology has led to the emergence of industrial wireless standards such as WirelessHART, WIA-PA and ISA100 wireless [6-9]. These standards are specifically designed for industrial monitoring and control applications in order to solve the problem of cumbersome cabling.

The WirelessHART, being based on the traditional HART protocol, has an edge over its counterpart with millions of HART-enabled devices already installed globally. However, applying the technology for control comes with the challenges of lack of adequate infrastructures such as WirelessHART adaptors. In an attempt to solve the problem of interfacing the WirelessHART network to the field elements such as sensors, transducers and actuators, Some prominent companies in the field, like EMERSON, AWIATECH, invested huge amount of money to create their own solution of wireless system between control plants, but these products are usually very expensive and are having proprietary issues. In a related development, a simple and inexpensive WirelessHART Adaptor was developed in [10] for process monitoring. 
Therefore, in this paper, the developed adaptor is extended for implementation on a flow control. For this purpose, few controllers including those proposed in [11], [12] and [13] will be implemented for flow control on a Pilot Plant.

The rest of the paper is organized as follows: In Section 2, the brief on the complete WirelessHART control set-up including interfaces is given. Section 3 gives the brief of the compared controllers while Section 4 presents and discusses the results. Lastly, conclusion is provided in Section 5.

\section{WIRELESSHART FLOW CONTROL LOOP SET-UP}

This section will first describe the complete experimental set-up including the process and instrumentation (P\&ID) diagram of the plant. Then, brief description of the adaptors/interface will be given at the second part.

\subsection{Complete System Set up}

The selected controllers will be implemented on a PcA SimExpert Flow Control and Calibration Process Mobile Pilot plant stationed at Block 23, Universiti Teknologi PETRONAS. The complete experimental set-up is shown in Figure 1 while the block diagram representation is given in Figure 2. Furthermore, the P\&ID of the plant is shown in Figure 3. The plant consist of a buffer tank and a calibration tank that are connected in series. The objective of the plant is to transfer fluid from the buffer tank to the calibration tank at a controlled flow rate. To achieve this, two pumps and a pneumatic valve are used. In this experiment, the measured value is water level while the manipulated value is the flow rate in $\mathrm{m} 3 / \mathrm{s}$. As seen in the figure, PIC110C is the main feedback controller while FT110C and CV110 are the flow transmitter and the control valve respectively. P101 and P201 are the pumps for buffer tank VE100 and calibration tank VE200 respectively. In the same vein, LS101 and LS201 are the respective level sensors connected to P101 and P201 for the control of overflow.

To achieve wireless control of the flow rate by controlling the opening of CV110 and to obtain flow measurement, the controller is implemented in Simulink environment in the host computer interfaced with Python to export the control action into the gateway. The control signal or manipulated variable (MV) is now received by the valve through a developed WirelessHART Adaptor. Measurements based on the process variable (PV) are received from the sensor via WirelessHART adaptor into the gateway and then the controller.

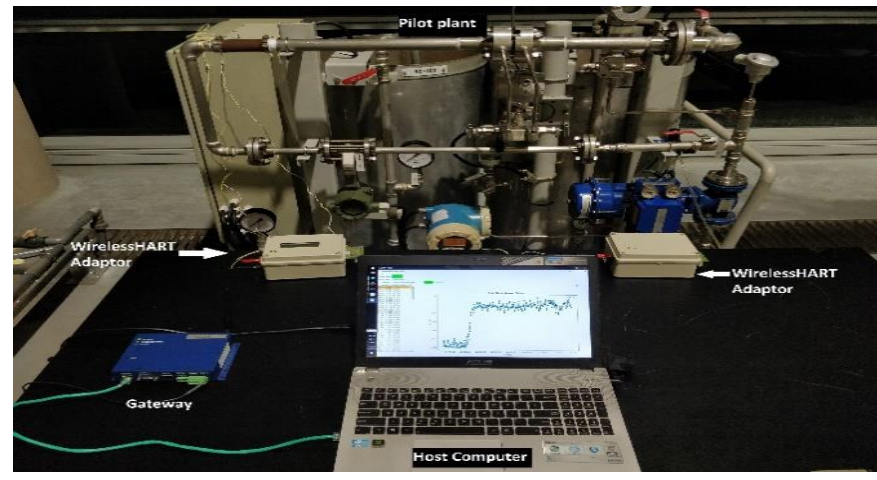

Figure 1. Complete experimental set-up

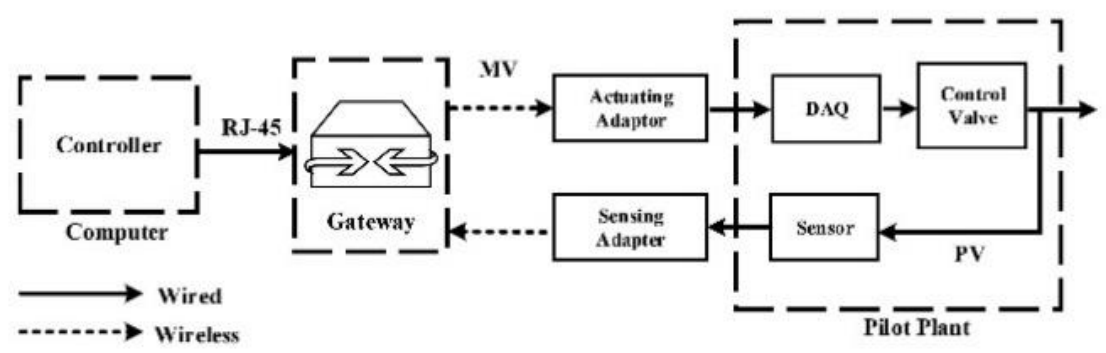

Figure 2. Block diagram representation of the experimental set-up 


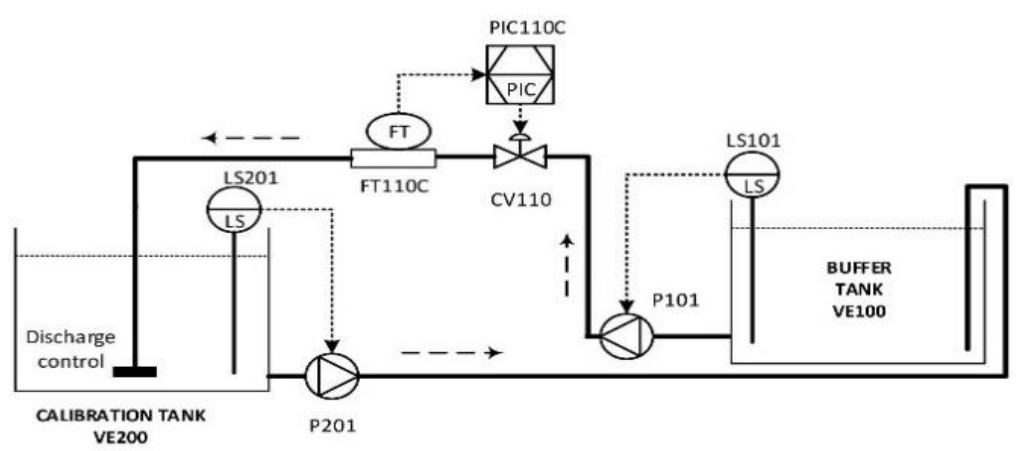

Figure 3. Simplified P\&ID diagram

\subsection{Adaptors / System Interface}

\subsubsection{WirelessHART Sensing Adaptor}

As shown in the block diagram of Figure 4, the sensing module is primarily formed by Arduino MEGA 2560 micro-controller board and a WirelessHART mote (DC9003A-C) from Linear Technology. In addition, an LCD display is added to the design in order to display real time sensor reading. This can be seen in the circuit implementation of the adaptor given in Figure 5. The pin-out connections between the microcontroller and the WirelessHART mote is given in Table 1.

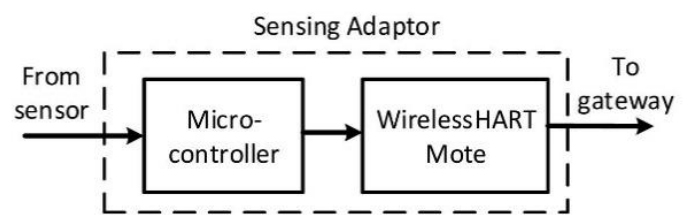

Figure 4. Block diagrams of Sensing WirelessHART adaptor

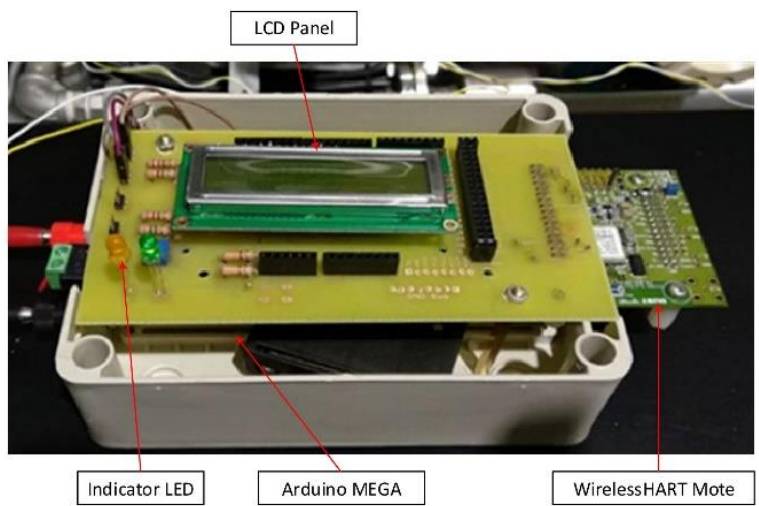

Figure 5. Sensing WirelessHART adaptor

Table 1. Mote/Micro-controller pin-out

\begin{tabular}{cc}
\hline DC9003A-C & Mega 2560 \\
\hline GND & GND \\
VBAT & $3.3 \mathrm{~V}$ \\
RX & TX1 \\
TX CTSn & GND \\
RX CTSn & $3.3 \mathrm{~V}$ \\
\hline
\end{tabular}




\subsubsection{WirelessHART Actuation Adaptor}

Similar to the sensing module, the actuating module is formed by Arduino MEGA 2560 microcontroller board and a WirelessHART mote (DC9003A-C). In addition, a 4-20mA T click board is used to convert the signal from the micro-controller into a 4-20mA signal. The main function of this module is to channel the control action generated by the host application to the valve in order to adjust the degree of actuation. Block diagrams as well the circuit implementation of the adaptor are shown in Figure 6 and 7 respectively. Furthermore, the pin-out connections between the micro-controller and the WirelessHART mote is similar to that of sensing adaptor and is given in Table 2.

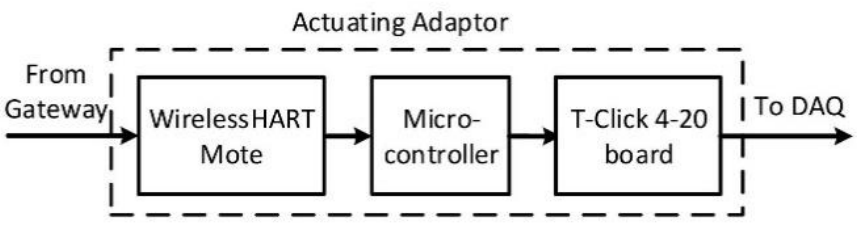

Figure 6. Block diagrams of Actuating WirelessHART adaptor

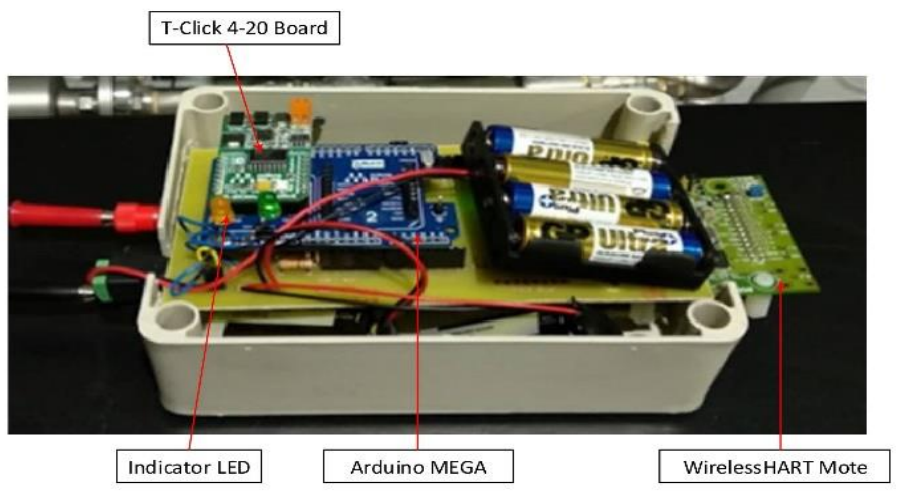

Figure 7. Actuating WirelessHART adaptor

The control valve in the plant is the Spirax Sarco pneumatic valve. Thus, the plant is equipped with a current to pressure converter (I/P Converter) that converts the 4-20mA current into equivalent pneumatic pressure value. The details of the I/P converter are shown in Table 2.

Table 2. I/P Converter Specifications

\begin{tabular}{cc}
\hline Spirax Sarco EP5 & Electro pneumatic Positioner \\
\hline Input Signal & $4-20 \mathrm{~mA}$ \\
Output Signal Range & $0-100 \%$ supply air pressure \\
Supply air pressure & $1.4-6.0 \mathrm{bar}$ \\
Voltage Rating & $5 \mathrm{~V}$ (Min) 24V (Max) \\
\hline
\end{tabular}

\section{CONTROL STRATEGIES}

This section will briefly discuss the controllers to be compared. The controllers compared are PI, Smith predictor, Fuzzy PID, Setpoint Weighting and Filtered Predictive PI. Some of these controllers are reported in our earlier works in $[11,13]$. For easy understanding of the controllers, consider the single loop WirelessHART network shown in Fig. 8. To facilitate analysis, the delays can be lumped as total networkinduced delay $\tau_{N}$ as given in (1).

$$
\tau_{N}=\tau_{C A}+\tau_{S C}
$$


where $\tau_{C A}$ and $\tau_{S C}$ are the controller-to-actuator and sensor-to-controller delays respectively, $G(s)=G_{n}(s) e^{-s L_{P}}$ is the plant model. Assuming there is commutativity, the process deadtime $\left(L_{P}\right)$ as given in the figure can be added to the network delay which now gives the total closed loop delay as in (2). These equations will be required for the design of the controllers.

$$
L=\tau_{N}+L_{P}
$$

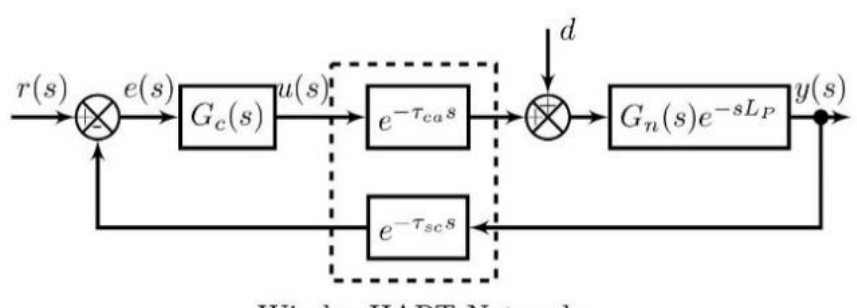

WirelessHART Network

Figure 8. Network delay representation in a single loop WirelessHART networked control system

\subsection{PI, Smith Predictor and Fuzzy PID}

The controller structure used for both PI and Smith predictor [14] is given in (3).

$$
G_{c}(s)=K_{c}\left(1+\frac{1}{T_{i} s}\right)
$$

where $K_{c}$ and $T_{i}$ are the controller gain and time constants respectively. On the other-hand, the Fuzzy PID control structure adopted in this work is shown in Figure 9.

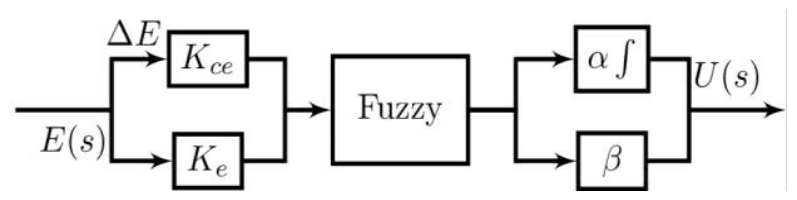

Figure 9. Fuzzy PID Structure

As seen from the structure, the controller has two inputs (error and change in error) and one output. The input scaling factors (SFs) $K_{e}$ and $K_{c e}$ are the respective error and error change gains, while the output SFs $\alpha$ and $\beta$ are the control gains. The output $U(s)$ of the controller is given in (4):

$$
u(t)=K_{p}+K_{i} \int e(t) d t+K_{d} \frac{d e(t)}{d t}
$$

In (4), Kp, Ki, and $\mathrm{Kd}$ are the proportional, integral, and derivative constants respectively. They are related to the fuzzy PID gains as $K_{p}=\alpha K_{c e}+\beta K_{e}, K_{i}=\alpha K_{e}, K_{d}=\beta K_{c e}$. Where, Ke is the input error scaling factor, Kce is the input error change scaling factor, $\alpha$ and $\beta$ are the output scaling factors

\subsection{Filtered Predictive PI (FPPI)}

The FPPI structure is given in shown in Figure 10. The difference between the conventional PPI structure and the FPPI is the inclusion of the filter term F(s) which will help curtail the effect of noise and oscillation induced by higher order systems and stochastic nature of the network. It should be noted that the design of PPI is based on FOPDT systems [15-16]. 


$$
U(s)=\left(K_{c} E(s)+\frac{1}{1+s T_{i}} e^{-s L} U(s)\right) F(s)
$$

where $F(s)$ is a filter transfer function.

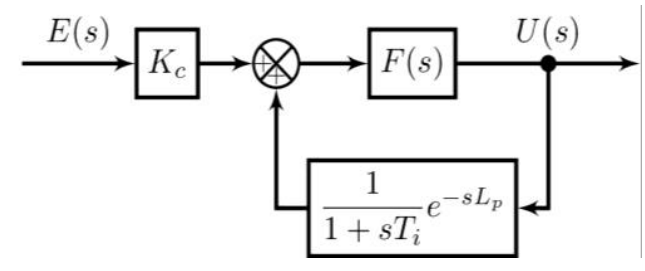

Figure 10. Implementation of FPPI controller

\subsection{Setpoint Weighting (SW)}

Consider the reference signal $\mathrm{r}(\mathrm{s})$ of Figure 8, using setpoint weighting function $\mathrm{fr}(\mathrm{s})$ of (6), this reference signal is varied from $\mathrm{r}(\mathrm{s})$ to $\tilde{r}(s)$. The implementation of (6) is shown in Figure 11. This allows for the 2-DoF ability of both good setpoint tracking and disturbance rejection of the controller. The complete design procedure for this approach can be found in our earlier work reported in [13, 17-21].

$$
f_{r}(s)=\frac{\tilde{r}(s)}{r(s)}=G_{r}(s)+\tilde{G}_{y r}(s)\left(e^{\tilde{r} s}-G_{r}(s)\right)
$$

where $\mathrm{Gr}(\mathrm{s})$ is the setpoint regulating feed-forward controller.

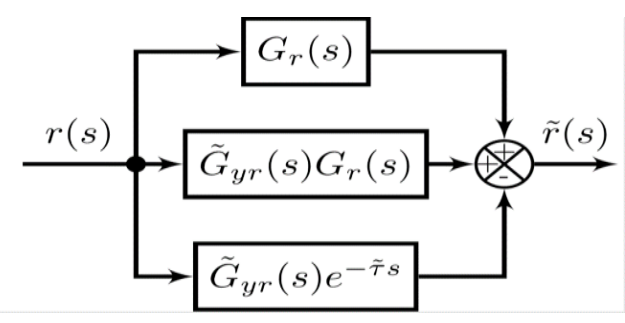

Figure 11. Structure of the general SW function fr(s)

\section{RESULTS AND ANALYSIS}

This section is divided into two parts. In the first part, various controller parameters and flow process plant model will be presented while the second part of the section will present the result comparison.

\subsection{Plant model and controllers Parameters}

The controllers were tested on a pilot flow control system of Figure 1. The model of the system is given in the transfer function of (7). The model was obtained using empirical modelling of the plant similar to that reported in [22] and [23]. The various controller parameters for the plant are given in Table 3. The Fuzzy PID and FPPI parameters were obtained through tuning with optimization algorithm on the model of the plant. Furthermore, the rule base table for the fuzzy PID based on [24] and [25] is given in Table 4. The definitions of the entries to the table are given as: Z for "Zero" while NS, NM, and NB stands for "Negative small", "Negative medium" and "Negative big" respectively. Others are PS, PM and PB which stands for "Positive small", "Positive medium" and "Positive big" respectively.

$$
G=\frac{0.58}{0.26 s+1} e^{-0.25 s}
$$

\subsection{Result Comparison}

In this experiment, the data sampling time in MATLAB is set as 0.5 seconds while the update rate between the gateway and field devices (motes/adaptors) is set as the $4 \mathrm{~s}$ which is at the middle of $1 \mathrm{~s}$ for fast 
piping and 8s for optimal battery performance. The disadvantage of the fast piping is that it drains the batteries of field devices faster. The duration of the experiment for each controller tested is 500 seconds. This is due to the capacity of the tanks VE100 and VE200. The target was set at $1.5 \mathrm{~m} 3 /$ hour. It should be noted that the valve position at $100 \%$ delivers a maximum of $2.5 \mathrm{~m} 3 /$ hour.

Table 3. Various controller parameters

\begin{tabular}{cc}
\hline Controller & Parameters \\
\hline PI \& Smith & $4-20 \mathrm{~mA}$ \\
FPPI & $0-100 \%$ supply air pressure \\
SW & $1.4-6.0$ bar \\
Fuzzy PID & $5 \mathrm{~V}$ (Min) 24V (Max) \\
\hline
\end{tabular}

Table 4. Fuzzy PID rule base

\begin{tabular}{cccccccc}
\hline E $\triangle \mathrm{E}$ & NB & NM & NS & Z & PS & PM & PB \\
\hline PB & Z & PS & PM & PB & PB & PB & PB \\
PM & NS & Z & PS & PM & PB & PB & PB \\
PS & NM & NS & Z & PS & PM & PB & PB \\
Z & NB & NM & NS & Z & PS & PM & PB \\
NS & NB & NB & NM & NS & Z & PS & PM \\
NM & NB & NB & NB & NM & NS & Z & PS \\
NB & NB & NB & NB & NB & NM & NS & Z \\
\hline
\end{tabular}

Result comparison of the PI, FPPI, SW, Fuzzy PID and Smith predictor controllers is shown in Fig. 12. The numerical information of the result as obtained from the figure is given in Table 5. From the figure, it can be clearly seen that FPPI controller has faster response with a rise time of around 34s. This is followed by PI and SW with $46.77 \mathrm{~s}$ and $56.22 \mathrm{~s}$ each. The slowest are the fuzzy and Smith predictor with respective rise times of 72.27 and $164.73 \mathrm{~s}$. Although the smith predictor recorded the least overshoot of $6.7 \%$, it is however very slow in response and experiences glitch at around 150s during the experiment. Comparing overshoots of the controllers against the PI, it can be seen that there is a significant difference between the $31.33 \%$ of the PI and the 6.7, 11.33, 14.67 and $16 \%$ of the Smith Pred., SW, Fuzzy and FPPI respectively. In a nutshell, all the other controllers have outperformed the PI in terms of overshoot while the FPPI has an added advantage of rise time over the PI.

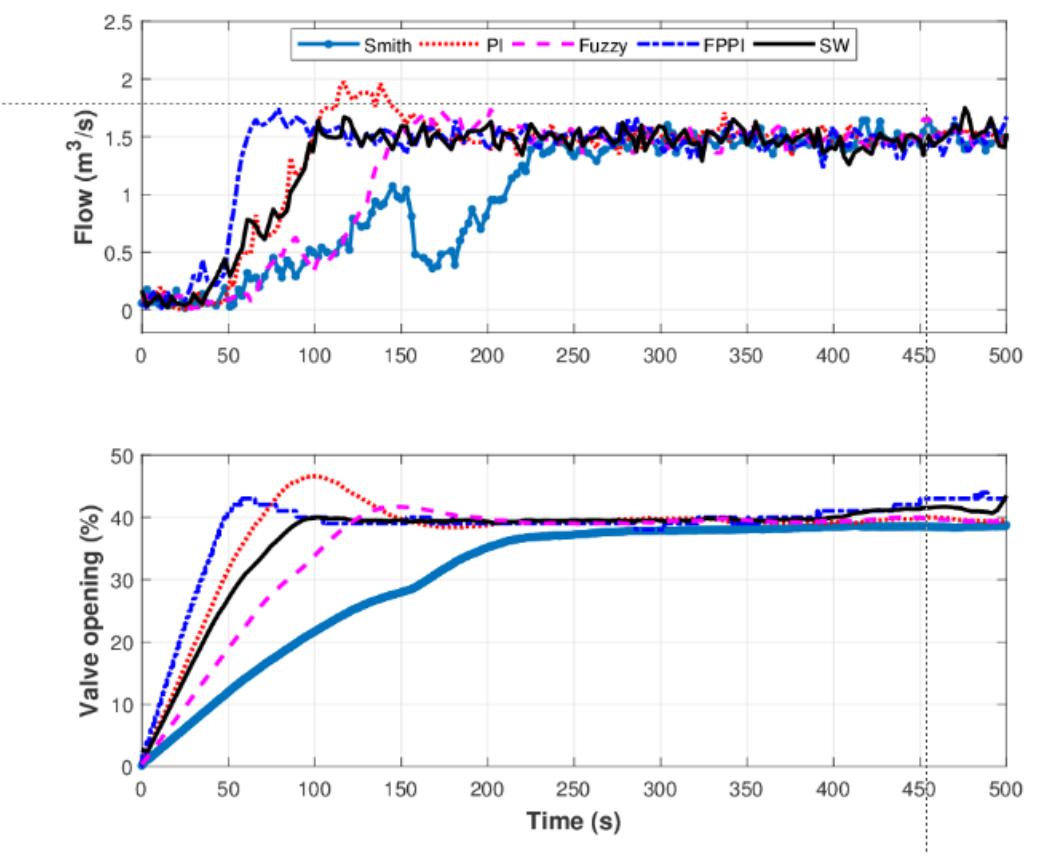

Figure 12. Comparison of various developed controllers for flow control 
Table 5. Performance of controllers for pilot plant

\begin{tabular}{ccc}
\hline \multirow{2}{*}{ Controller } & \multicolumn{2}{c}{ System's Performance } \\
& Rise Time $(\mathrm{s})$ & Overshoot $(\%)$ \\
\hline SW & 56.22 & 11.33 \\
FPPI & 33.95 & 16.00 \\
Fuzzy PID & 72.27 & 14.67 \\
PI & 46.77 & 31.33 \\
Smith Pred. & 164.73 & 6.70 \\
\hline
\end{tabular}

The respective characteristics of each controller as seen from its response is also manifested through the control signals. While the signal of FPPI rose steadily to $40 \%$ in less than 50 s, the Smith predictor is the most sluggish by reaching the $40 \%$ mark at around 200s. It should also be noted that, the signal of SW despite being slower than both FPPI and PI, does not go beyond the $40 \%$ position of the valve as compared to the FPPI, PI and Fuzzy PID. This is responsible for its lower overshoot compared to the Fuzzy PID, FPPI and PI. Thus, after comparing all the results, it can be succinctly stated that the SW, FPPI and fuzzy PID controllers have improved on both the PI and Smith predictor controllers.

\section{CONCLUSION}

In this paper, the use of WirelessHART adaptors for control has been demonstrated. The sensing and actuating adaptors employed here were developed locally using inexpensive components. Thus, these adaptors permitted the actualization of wireless control. Furthermore, experimental results of the controllers compared shows that despite the network induced delay, improved performance of the PI and Smith predictor approaches can be achieved through the use of FPPI, SW and fuzzy PID strategies.

\section{ACKNOWLEDGEMENTS}

This work was supported by Universiti Teknologi PETRONAS and Abubakar Tafawa Balewa University

\section{REFERENCES}

[1] Q. Wang and J. Jiang, "Comparative examination on architecture and protocol of industrial wireless sensor network standards," IEEE Communications Surveys \& Tutorials, vol. 18, no. 3, pp. 2197-2219, 2016

[2] N. Aslam, K. Xia, A. Ali, and S. Ullah, "Adaptive tcp-iccw congestion control mechanism for qos in renewable wireless sensor networks," IEEE Sensors Letters, vol. 1, no. 6, pp. 1-4, 2017.

[3] T. Gomes, S. Pinto, F. Salgado, A. Tavares, and J. Cabral, "Building ieee 802.15. 4 accelerators for heterogeneous wireless sensor nodes," IEEE Sensors Letters, vol. 1, no. 1, pp. 1-4, 2017.

[4] S. Kharb and A. Singhrova, "Review of industrial standards for wireless sensor networks," in Next-Generation Networks. Springer, 2018, pp. 77-87.

[5] I. J. Halvorsen, "Handling of variable wireless latency and updating frequency in pi controllers," IFACPapersOnLine, vol. 49, no. 7, pp. 336-341, 2016.

[6] Henk, B. V. D. "Full redundant field wireless automation solutions based on the isa100. 11a standard." Flow Level Pressure 2014 (2014): 14-15.

[7] Habib, Gilbert, Nicolas Haddad, and Ralph El Khoury. "Case study: WIRELESSHART vs ZIGBEE network" In 2015 Third International Conference on Technological Advances in Electrical, Electronics and Computer Engineering (TAEECE), pp. 135-138. IEEE, 2015.

[8] Flammini, Alessandra, Paolo Ferrari, Daniele Marioli, Emiliano Sisinni, and Andrea Taroni. "Wired and wireless sensor networks for industrial applications." Microelectronics Journal, no. 9 (2009): 1322-1336.

[9] Wang, Quan, and Jin Jiang. "Comparative examination on architecture and protocol of industrial wireless sensor network standards." IEEE Communications Surveys \& Tutorials 18, no. 3 (2016): 2197-2219.

[10] T. Supramaniam, R. Ibrahim, S. M. Hassan, and K. Bingi, "Development of wirelesshart adapter with industrial transmitter for process monitoring," in 2017 IEEE International Conference on Signal and Image Processing Applications (ICSIPA), 2017, pp. 294-298.

[11] S. M. Hassan, R. Ibrahim, N. Saad, V. S. Asirvadam, T. D. Chung, and S. Iskandar, "A filtered predictive pi controller for WirelessHART networked control system," Journal of Control Engineering and Applied Informatics, vol. 19, no. 4, pp. 13-24, 2017.

[12] S. M. Hassan, R. Ibrahim, N. Saad, V. S. Asirvadam, and T. D. Chung, "Setpoint weighted WirelessHART networked control of process plant," in Instrumentation and Measurement Technology Conference Proceedings (I2MTC), 2016 IEEE International. IEEE, 2016, pp. 1-6.

[13] S. M. Hassan, R. Ibrahim, N. Saad, V. S. Asirvadam, and K. Bingi, "Adopting setpoint weighting strategy for WirelessHART networked control systems characterized by stochastic delay," IEEE Access, vol. 5, pp. 25885-25896, 2017. 
[14] Bingi, Kishore, Rosdiazli Ibrahim, Mohd Noh Karsiti, Sabo Miya Hassan, and Vivekananda Rajah Harindran. "A comparative study of 2DOF PID and 2DOF fractional order PID controllers on a class of unstable systems." Archives of Control Sciences, 28(4), pp635-682, 2018.

[15] Segovia, V. Romero, Tore Hägglund, and Karl Johan Åström. "Measurement noise filtering for PID controllers." Journal of Process Control 24, no. 4 (2014): 299-313.

[16] Huba, Mikuláš, and Igor Bélai. "Experimental evaluation of a DO-FPID controller with different filtering properties." IFAC Proceedings Volumes 47, no. 3 (2014): 198-203.

[17] Alfaro, Víctor M., Ramon Vilanova, and Orlando Arrieta. "Considerations on set-point weight choice for 2-DoF PID controllers." IFAC Proceedings Volumes 42, no. 11 (2009): 721-726.

[18] Bianchi, F.D., Mantz, R.J. and Christiansen, C.F., 2008. Multivariable PID control with set-point weighting via BMI optimisation. Automatica, 44(2), pp.472-478.

[19] Mudi, R.K. and Dey, C., 2011. Performance improvement of PI controllers through dynamic set-point weighting. ISA transactions, 50(2), pp.220-230.

[20] YEŞiL, E., Guezelkaya, M., Eksin, I. and Tekin, O.A., 2008. Online tuning of set-point regulator with a blending mechanism using PI controller. Turkish Journal of Electrical Engineering \& Computer Sciences, 16(2), pp.143157.

[21] Yesil, E., Kaya, M. and Siradag, S., 2012, July. Fuzzy forecast combiner design for fast fashion demand forecasting. In 2012 International Symposium on Innovations in Intelligent Systems and Applications (pp. 1-5). IEEE.

[22] T. A. H. AL-Qutami and R. Ibrahim, "Design of a fuzzy logic process controller for flow applications and implementation in series tanks pilot plant," in Industrial Instrumentation and Control (ICIC), 2015 International Conference on. IEEE, 2015, pp. 450-455.

[23] Chien, Lim Jin, Rosdiazli Ibrahim, Sabo Miya Hassan, Kishore Bingi, and ThasarathaRao Supramaniam. "Comparison Between Wired and WirelessHART Networked PID Control of Process Flow." In 2018 International Conference on Intelligent and Advanced System (ICIAS), pp. 1-5. IEEE, 2018.

[24] Duan, Xiao-Gang, Hua Deng, and Han-Xiong Li. "A saturation-based tuning method for fuzzy PID controller." IEEE Transactions on Industrial Electronics 60, no. 11 (2013): 5177-5185.

[25] Pan, Indranil, Saptarshi Das, and Amitava Gupta. "Tuning of an optimal fuzzy PID controller with stochastic algorithms for networked control systems with random time delay." ISA transactions 50, no. 1 (2011): 28-36.

\section{BIOGRAPHIES OF AUTHORS}
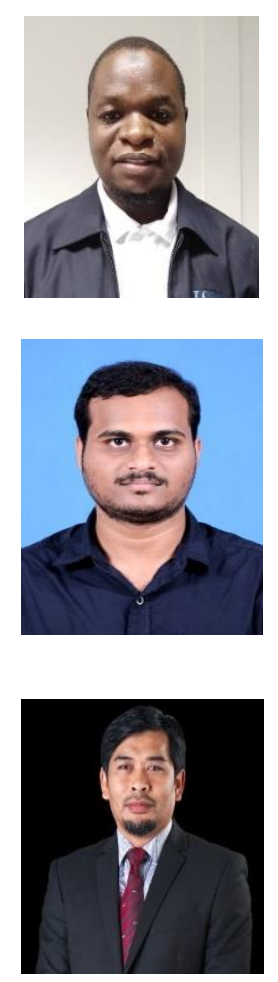

Sabo Miya Hassan received the B.Eng. degree (Hons.) in electrical and electronic engineering from Abubakar Tafawa Balewa University, Bauchi, Nigeria, in 2008, the M.Sc. Eng. Degree (Hons.) in control systems from the University of Sheffield, U.K., in 2011, and the Ph.D. degree in Electrical and Electronic Engineering from the Universiti Teknologi Petronas, Perak, Malaysia in 2018. He is currently with the Department of Electrical and Electronic Engineering, Abubakar Tafawa Balewa University, Bauchi, Nigeria and Kabale University Uganda. His current research interests include wireless networked control systems, intelligent control, soft computing, and optimization.

Kishore Bingi is a Research Scientist in the Department of Electrical \& Electronic Engineering at Universiti Teknologi PETRONAS (UTP), Malaysia. He obtained his B.Tech. degree in Electrical and Electronics Engineering from Bapatla Engineering College, Andhra Pradesh, India, in 2012, and the M.Tech. degree in Instrumentation and Control Systems from National Institute of Technology Calicut (NITC), Kerala, India, in 2014. He worked with TATA Consultancy Service (TCS) as an Assistant Systems Engineer from 2014 to 2015. He is currently pursuing the Ph.D. degree in the Department of Electrical and Electronic Engineering, Universiti Teknologi PETRONAS (UTP), Perak, Malaysia. His current research interests include nonlinear process modeling, fractional-order control and optimization.

Rosdiazli Ibrahim received the B.Eng. degree in electrical engineering from Universiti Putra Malaysia, Kembangan, Malaysia, in 1996, the M.Sc. degree in automation and control from Newcastle University, Newcastle upon Tyne, U.K., in 2000, and the Ph.D. degree in electrical and electronic engineering from the University of Glasgow, Glasgow, U.K., in 2008. He is an Associate Professor with the Department of Electrical and Electronics Engineering at Universiti Teknologi PETRONAS (UTP), Seri Iskandar, Perak, Malaysia. He is currently, The Dean, Centre for Graduate Studies, UTP. His current research interests include intelligent control and non-linear multi-variable process modeling for control application. 


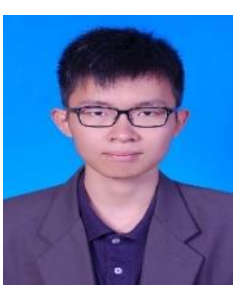

Lim Jin Chien received B..Eng. degree (Hons.) in Electrical and Electronics Engineering from Petronas Universiti Teknologi Petronas (UTP), Perak, Malaysia in 2018. He is currently an Instrument and Control Engineer in Engineering and Technical Services Department, Gas Processing and Utilities Segment of Petronas Gas Berhad, Malaysia.

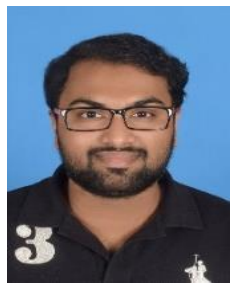

THASARATHARAO received the B.Eng. (Hons.) degree in Electrical \& Electronics Engineering from Universiti Teknologi Petronas (UTP), Seri Iskandar,Perak, Malaysia in 2016. He is currently pursuing the M.Sc. (Hons.) degree with the Electrical and Electronic Engineering Department, Universiti Teknologi PETRONAS (UTP), Perak, Malaysia. His current research interest include wireless networked control system, internet of everything (IoT), intelligent control and artificial intelligence. 